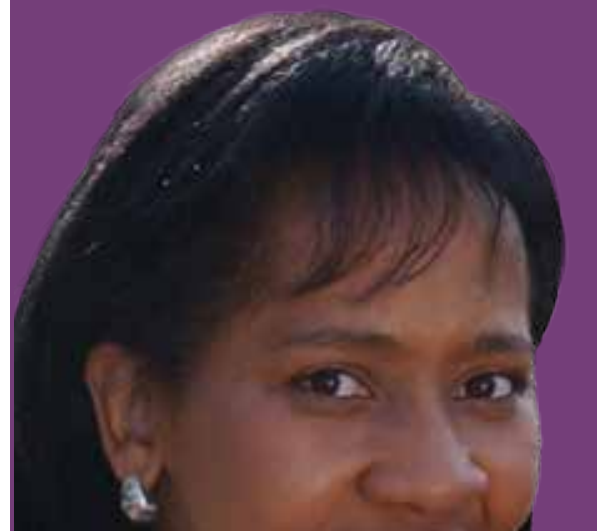

\title{
Weight Gain and Pregnancy
}

Many factors are used to determine the right amount of weight to gain during pregnancy. Discuss with your doctor and keep a record of weight gained each time you visit the doctor.

Everything you eat and drink matters. Make healthy choices every day to stay healthy and have a healthy, full term baby.

- Drink water and low fat or nonfat milk.

- Eat a variety of vegetables.

- Choose whole fruit over juice.

- Use whole grain breads and cereals.

\section{Did You Know:}

Your baby can taste some of the flavors of the foods you eat during pregnancy. That's one reason to eat smart!

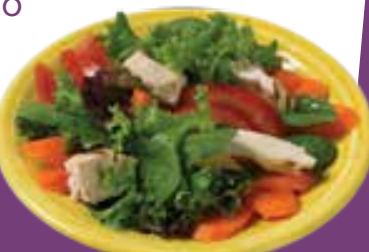

- Eat lean sources of protein foods.

- Drink and eat less sodium, saturated fat, and added sugars.

\section{If before pregnancy you are... \\ During pregnancy you should gain...}

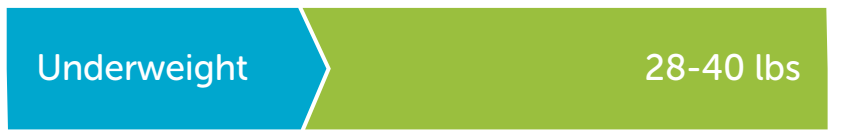

Normal (healthy) weight

25-35 lbs

Overweight $\quad$ 15-25 lbs

Obese

$11-20$ lbs

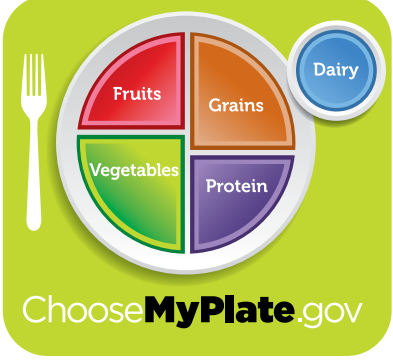

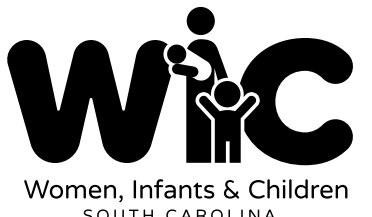

SOUTH CAROLINA 


\section{Where Does it Go?}

In addition to your growing belly, you may have noticed the rest of your body changing. Weight gained during pregnancy is not just the baby; you will have weight gain all over your body to help your growing baby. The picture shows a woman who gained a healthy amount of weight during pregnancy.

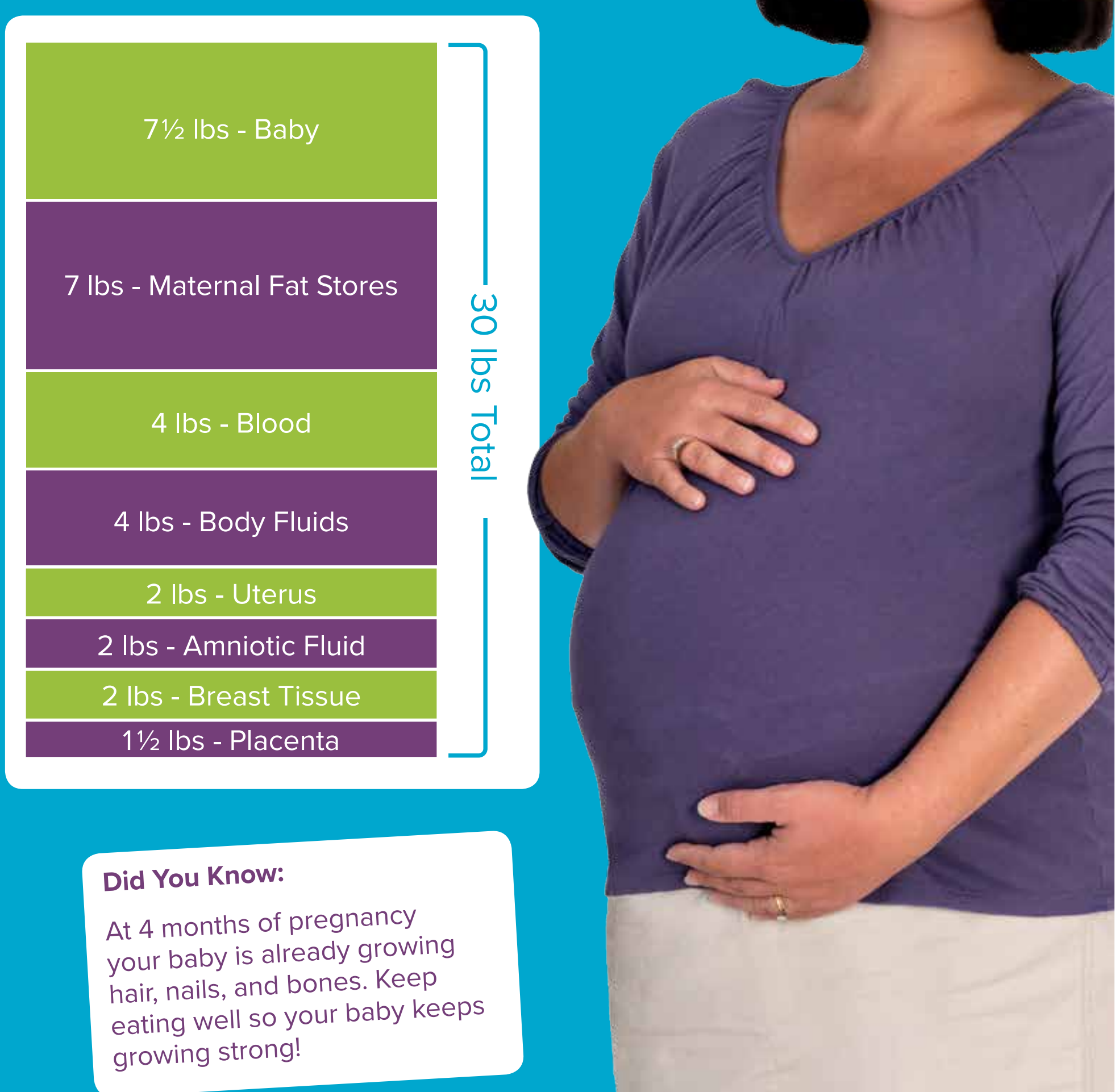

